\title{
Ubicación de Generación Distribuida para Minimización de Pérdidas Usando un Algoritmo Genético Híbrido
}

\author{
Pablo A. Narváez ${ }^{(1)}$, Jesús M. López-Lezama(2) y Esteban Velilla(2) \\ (1) Interconexión Eléctrica S.A. (ISA), Calle 12Sur No. 18-168, Medellín - Colombia \\ (e-mail: pablonb@gmail.com). \\ (2) Grupo de Investigación GIMEL, Departamento de Ingeniería Eléctrica, Facultad de Ingeniería, \\ Universidad de Antioquia, Calle 70 No. 52-21, Medellín - Colombia (e-mail: lezama@udea.edu.co; \\ esteban.velilla@gmail.com)
}

Recibido Jul. 31, 2014; Aceptado Oct. 21, 2014; Versión final recibida Oct. 29, 2014

\section{Resumen}

En este artículo se presenta una metodología para la ubicación óptima de Generación Distribuida (GD) usando un algoritmo genético híbrido. En este caso la función objetivo es la reducción de pérdidas. El algoritmo propuesto incorpora una red neuronal artificial para evaluar la función de adaptación y una búsqueda local que permite al algoritmo explorar un espacio de búsqueda más amplio. La contribución principal del artículo es la combinación de técnicas metaheurísticas con técnicas de inteligencia artificial para resolver un problema de optimización multi-modal y no convexo. La metodología propuesta es probada en un sistema de 34 barras mostrando que la ubicación apropiada de las unidades de GD permite reducir las pérdidas activas y mejorar el perfil de tensiones.

Palabras clave: algoritmos genéticos, generación distribuida, redes neuronales artificiales

\section{Location of Distributed Generation for Power Losses Reduction using a Hybrid Genetic Algorithm}

\begin{abstract}
This paper presents a methodology for optimal Distributed Generation (DG) allocation using a hybrid genetic algorithm. In this case, the objective function is the minimization of power losses. The proposed algorithm involves an artificial neural network to evaluate the fitness function and a local search subroutine that allows the algorithm to explore a wider search space, and eventually, escape from local optimal solutions. The proposed approach was tested on a 34-bus system showing that the proper allocation of DG units allows the reduction of active power losses and the improvement of voltage profile.
\end{abstract}




\section{INTRODUCCIÓN}

La Generación Distribuida (GD) se entiende como la producción de electricidad a pequeña escala realizada directamente en la red de distribución o en las instalaciones del usuario final. Para la producción de electricidad, la GD puede utilizar fuentes de energía renovable (generación eólica, solar, biodiesel) o no renovable (generación con turbinas, microturbinas, motores de combustión. Dada su ubicación cerca de la demanda, la GD puede contribuir a la disminución de pérdidas activas y al mejoramiento del perfil de tensiones (Porkar et al., 2010). Adicionalmente, estudios recientes han mostrado que la GD puede traer otros beneficios como el aplazamiento de inversiones en la red (Gautam \& Mithulananthan, 2007), el aumento en la confiabilidad (Jabr y Pal 2009) y la reducción de congestión (Liu et al. 2011). Sin embargo, los beneficios de la GD dependen en gran medida de su ubicación y dimensionamiento. Se ha demostrado también que una inadecuada planeación de la GD puede traer efectos negativos al sistema como el aumento de pérdidas y sobretensiones en la red (Borges y Falcão 2006). Por esta razón, es de vital importancia contar con metodologías que permitan determinar la ubicación apropiada de GD para obtener sus máximos beneficios y al mismo tiempo, respetar las restricciones operativas de la red. En este contexto, existen diferentes metodologías reportadas en la literatura técnica especializada que buscan encontrar la mejor ubicación de GD en las redes de energía eléctrica. Estas metodologías incluyen métodos analíticos (Khan y Choudhry, 2010), (Hung, 2010) heurísticas(Hemdan y Kurrat, 2011; Ouyang et al., 2011) metaheurísticas (López-Lezama et al. 2012; Kang et al. 2012; Biswas et al. 2012) y métodos basados en programación matemática (Khalesi et al. 2011; Banerjee y Islam 2011).

Dada la naturaleza intrínseca no lineal, multi-modal (múltiples óptimos locales) y no convexa del problema de ubicación óptima de GD, los métodos basados en metaheurísticas han mostrado ser los más apropiados para abordar este tipo de problema, especialmente cuando no se consideran aproximaciones lineales en las ecuaciones de flujo de carga de la red. En este artículo se presenta una metodología para la ubicación óptima de GD basada en un Algoritmo Genético (AG), combinado con una Red Neuronal Artificial (RNA). El objetivo que se pretende es la minimización de pérdidas en la red. El AG es utilizado para determinar las posibles ubicaciones de las unidades de GD, mientras que la RNA es utilizada para calcular las pérdidas de potencia activa, evitando así el uso de software para calcular el flujo de carga. Adicionalmente, el AG cuenta con una subrutina de Búsqueda Local (BL) que se ejecuta en cada iteración para procurar mejores soluciones.

\section{FORMULACIÓN MATEMÁTICA}

Para la formulación del problema se ha optado por la versión AC del flujo de carga. La función objetivo y restricciones se describen a continuación.

\section{Función Objetivo}

La función objetivo consiste en la minimización de pérdidas y está dada por la ecuación (1):

$\operatorname{Min} \sum_{r=1}^{N R} I_{r}^{2} R_{r}$

Donde NR es el número de ramas de la red, $I_{r}$ es la corriente por la rama $r$ y $R_{r}$ es la resistencia de la rama $r$. Al instalar GD la demanda neta de la red se reduce y esto se puede reflejar en una reducción en las corrientes de las ramas y por lo tanto, en las pérdidas del sistema. Sin embargo, un sobredimensionamiento de la GD puede tener el efecto contrario.

\section{Restricciones de igualdad}

Las restricciones de igualdad están dadas por las ecuaciones de balance de potencia activa y reactiva, representadas por las ecuaciones (2) y (3), respectivamente.

$$
\begin{aligned}
& P_{G i}-P_{D i}-P_{i}(V, \theta)=0 \\
& Q_{G i}-Q_{D i}-Q_{i}(V, \theta)=0
\end{aligned}
$$

En que $P_{G i}$ y $Q_{G i}$ son las potencias activa y reactiva, respectivamente, entregadas por una unidad de GD si ésta fuera ubicada en la barra $i$. En este caso no todas las barras tendrán GD, de tal modo que para cada unidad de GD se asigna una variable binaria que toma un valor de 1 si la unidad está ubicada en la barra 
correspondiente y 0 en caso contrario. Por simplicidad, no se ha considerado que la GD inyecte o tome potencia reactiva de la red, por lo que $Q_{G i}=0$. $P_{D i}$ y $Q_{D i}$ corresponden a las demandas de potencia activa y reactiva, en la barra $i$, respectivamente. Finalmente, $P_{i}(v, \theta)$ y $Q_{i}(v, \theta)$ corresponden a las inyecciones netas de potencia activa y reactiva, en la barra $i$, respectivamente, calculadas mediante las expresiones (4) y (5).

$$
\begin{aligned}
& P_{i}(V, \theta)=V_{i} \sum_{k=1}^{n b}\left[V_{k}\left\{g_{i k} \cos \left(\theta_{i k}\right)+b_{i k} \operatorname{sen}\left(\theta_{i k}\right)\right\}\right] \\
& Q_{i}(V, \theta)=V_{i} \sum_{k=1}^{n b}\left[V_{k}\left\{g_{i k} \operatorname{sen}\left(\theta_{i k}\right)-b_{i k} \cos \left(\theta_{i k}\right)\right\}\right]
\end{aligned}
$$

Donde $n b$ es el número de barras, $\theta_{i k}$ es la abertura angular entre las barras $i$ y $k$; $g_{i k}$ y $b_{i k}$ son las partes real e imaginaria, respectivamente, de la matriz de admitancia nodal. En las restricciones de igualdad también se han incluido las expresiones que describen los flujos de potencia activa y reactiva en las líneas, como se indica en las ecuaciones (6) y (7), respectivamente:

$$
\begin{aligned}
& P_{i k}=V_{i}^{2} g_{i k}-V_{i} V_{k} g_{i k} \cos \left(\theta_{i k}\right)-V_{i} V_{k} b_{i k} \operatorname{sen}\left(\theta_{i k}\right) \\
& Q_{i k}=-V_{i}^{2} b_{i k}+V_{i} V_{k} b_{i k} \cos \left(\theta_{i k}\right)-V_{i} V_{k} g_{i k} \operatorname{sen}\left(\theta_{i k}\right)
\end{aligned}
$$

Finalmente, la magnitud de la potencia aparente se puede expresar en función de las potencias activa y reactiva como se indica en la ecuación (8).

$$
S_{i k}^{2}=P_{i k}^{2}+Q_{i k}^{2}
$$

\section{Restricciones de desigualdad}

Las restricciones de desigualdad deben considerar los límites de potencia inyectada por las unidades de GD, los límites de tensión en la red y los límites de flujo de carga como se indica en las ecuaciones (9), (10) y (11), respectivamente. En este caso los superíndices min y max indican los límites mínimos y máximos, respectivamente.

$$
\begin{aligned}
& P_{G j}^{\min } \leq P_{G j} \leq P_{G j}^{\max } \\
& V_{i}^{\min } \leq V_{i} \leq V_{i}^{\max } \\
& \left|S_{i k}\right| \leq S_{i k}^{\max }
\end{aligned}
$$

\section{METODOLOGÍA}

La estrategia implementada para la ubicación óptima de GD consiste en un AG híbrido que involucra una RNA y una Búsqueda Local. A continuación se describe en detalle la metodología implementada.

\section{Algoritmo Genético}

Los algoritmos genéticos son estrategias de búsqueda utilizadas para resolver problemas complejos. Estos algoritmos replican procesos biológicos que la naturaleza utiliza en el proceso evolutivo de las especies, favoreciendo a los individuos más aptos (Abou El-Ela et al. 2010). Los Algoritmos Genéticos han sido ampliamente utilizados en aplicaciones relacionadas con la industria eléctrica, como el planeamiento de la expansión (García et al., 2012) y la optimización de redes eléctricas (Anaut et al., 2009).

Para la codificación del problema se optó por una cadena de bits de unos y ceros (cromosoma). El algoritmo está codificado para ubicar como máximo 5 generadores en las barras del sistema. En la codificación se utilizaron 6 números binarios para ubicar cada unidad de GD en las barras del sistema. Esta estructura permitiría la codificación desde 0 hasta 63; consecuentemente, los números que no existen como barras en el sistema se corrigen al momento de evaluar la función objetivo. En la Tabla 1 se ilustra la codificación. En este caso se indica que hay GD en los nodos 1, 13, 9, 4 y 2. 
Tabla 1: Ejemplo de codificación.

\begin{tabular}{|c|c|c|c|c|c|}
\hline & GD1 & GD 2 & GD 3 & GD 4 & GD 5 \\
\hline BUS & 1 & 13 & 9 & 4 & 2 \\
\hline BINARIO & 000001 & 001101 & 001001 & 000100 & 000010 \\
\hline
\end{tabular}

Para ejecutar el AG se genera inicialmente, de forma aleatoria, una población inicial de $n$ individuos, cada uno con una estructura similar a la presentada en la Tabla 1. Para cada uno de los individuos de la población inicial se debe evaluar la función de adaptación, en este caso, las pérdidas del sistema. Para esto se utiliza una RNA que será explicada en la siguiente sección.

A partir de la población inicial, y basándose en su función de adaptación, se deben seleccionar los individuos que heredarán sus características a la siguiente generación. Para la selección de dichos individuos se implementó un torneo binario. Para la generación de nuevos individuos se recombina la información de los padres. En este caso se utilizó recombinación en un solo punto seleccionado aleatoriamente, como se ilustra en la Figura 1.Luego del proceso de recombinación, los individuos pasan a la etapa de búsqueda local.
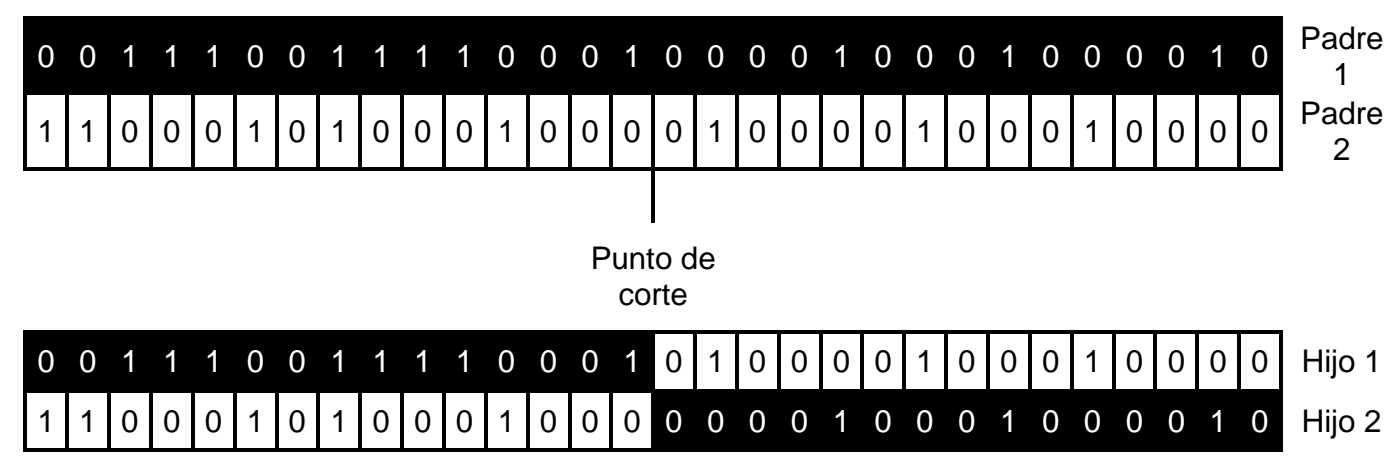

Fig1: Ejemplo de recombinación

\section{Búsqueda Local}

La Búsqueda Local evalúa posibles soluciones dentro de un vecindario previamente definido y selecciona la mejor solución de dicho vecindario. Para el sistema seleccionado, para cada iteración del AG se generó un vecindario cambiando un bit de la solución en cada una de las posiciones del cromosoma. La principal ventaja de la Búsqueda Local es que permite encontrar soluciones de alta calidad en las primeras iteraciones del algoritmo, lo que favorece la convergencia del mismo. En la Figura 2 se ilustra el flujograma de la metodología implementada.

Con el fin de calcular las pérdidas de potencia activa del sistema en el cual se desea ubicar la generación distribuida, una RNA es entrenada, validada y probada. A través de la RNA se evalúa la función objetivo que consiste en minimizar las pérdidas del sistema en cuestión. Una descripción detallada de esta RNA puede ser consultada en (Narváez, 2014). En este caso la red neuronal permite estimar las pérdidas del sistema para cada uno de los individuos de la población, evitando el cálculo del flujo de carga mediante métodos convencionales.

\section{PRUEBAS Y RESULTADOS}

Para validar la metodología propuesta se utilizó el sistema de 34 barras ilustrado en la Figura 3 . Este sistema es similar en topología al sistema IEEE de 34 barras, pero en una versión monofásica. Los datos de este sistema se encuentran disponibles en (Arroyave \& Lezama, 2013). La distribución de carga en los nodos se ilustra en la Figura 4. Se puede observar que gran parte de la demanda se encuentra ubicada lejos de la subestación. Por simplicidad se asume que todos los nodos son aptos para recibir GD, pero el número de unidades de GD se ha limitado a 5. Para el problema bajo estudio se definió como restricción un número máximo de una unidad de GD por barra con una capacidad de $1 \mathrm{MW}$. En la Tabla 2 se presenta en resumen los parámetros usados en el AG. 


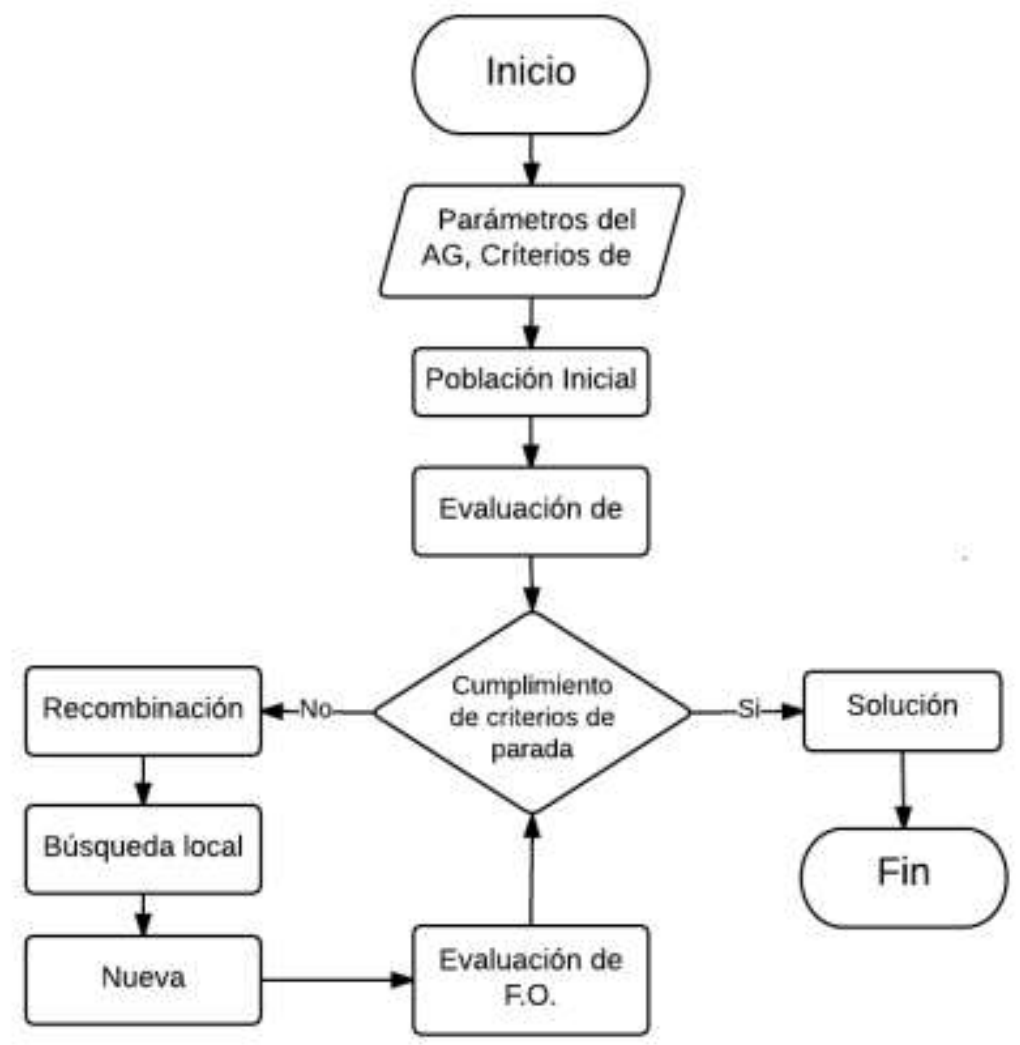

Fig.2: Diagrama de flujo de la metodología implementada.

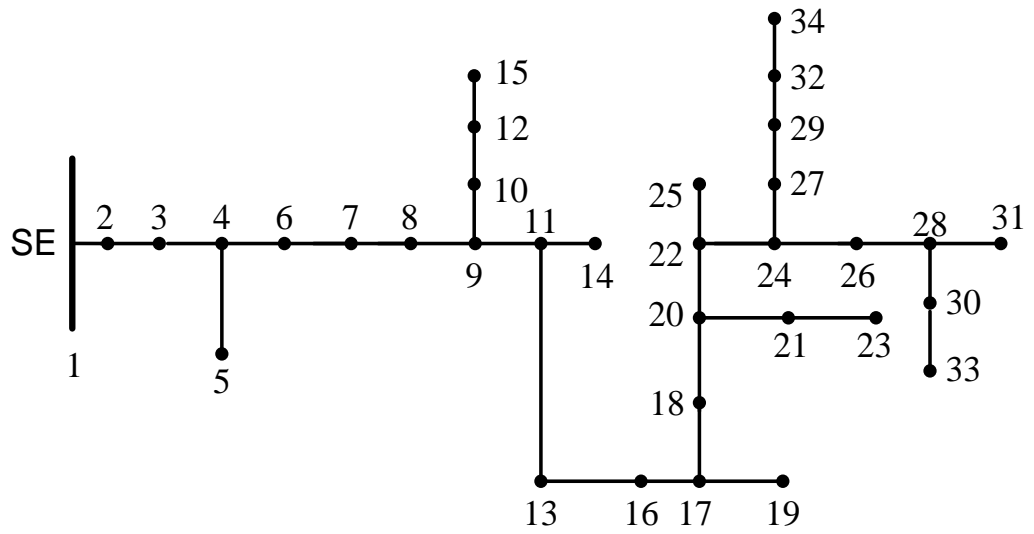

Fig. 3: Sistema de prueba de 34 barras

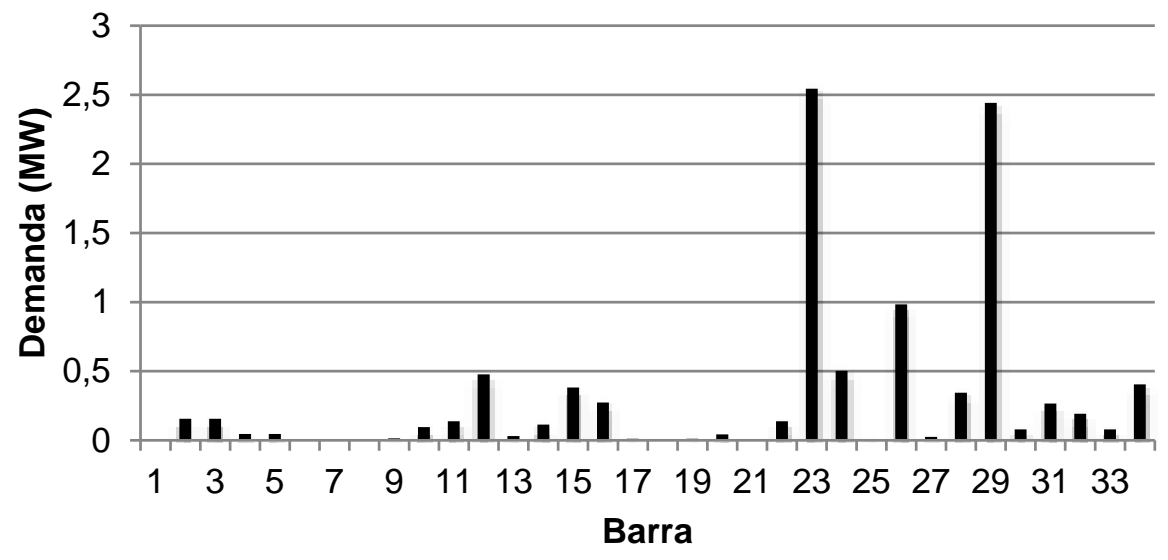

Fig. 4: Distribución de la demanda en la red de prueba 
Tabla 2: Parámetros del AG

\begin{tabular}{|l|c|}
\hline Parámetro & Valor \\
\hline Tamaño de la población & 20 \\
\hline Número máximo de generaciones & 40 \\
\hline Número de cortes para cruce & 1 \\
\hline Probabilidad de cruce & 0,95 \\
\hline
\end{tabular}

Para evaluar las pérdidas se seleccionó una red neuronal de dos capas feed-foward con una capa de oculta de función sigmoide y una capa de salida con una función linear. La capa oculta está constituida por 20 neuronas y la capa de salida por una sola. La red fue entrenada con el algoritmo Backpropagation de Levenberg-Marquardt, con una taza de aprendizaje de 0,01. En la Figura 5 se detalla la estructura de la red utilizada.

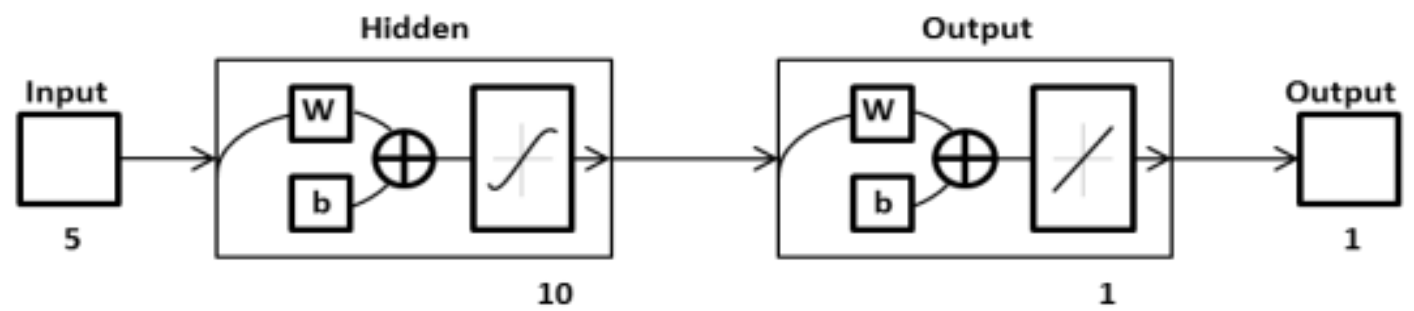

Fig. 5: Estructura de la RNA

Para entrenar la RNA se consolidó una base de datos de 2280 casos. Se pudo comprobar que una vez entrenada, la RNA entrega valores de pérdidas de la red muy similares a los obtenidos con un programa de flujo de carga convencional (Zimmerman 2011). Para el control de las soluciones infactibles, la RNA penaliza las soluciones con pérdidas altas buscando que el AG descarte de forma natural este individuo, evitando que aparezca en futuras iteraciones.

En la Figura 6 se ilustra una muestra de la plantilla de la base de datos usada para el entrenamiento de la RNA. El $70 \%$ de las muestras fueron seleccionadas para ser usadas en el entrenamiento, el $15 \%$ para la validación y el restante para la prueba de la RNA. Esto permite que la red no se limite a memorizar valores de los propuestos, sino también a intuir y a entregar valores bajo la inferencia de los valores intermedios.

Los resultados del entrenamiento con la red seleccionada muestran una respuesta adecuada, como se puede apreciar en la Tabla 3 y en la Figura 7. El EMC (Error Medio Cuadrático) corresponde a la diferencia promedio del cuadrado de los resultados y los objetivos de la red neuronal, valores bajos son mejores y un cero significa que no hay error. La R (Regresión R) es la correlación entre los resultados y los objetivos. Un valor de $\mathrm{R}$ igual a 1 indica una relación cercana, el 0 una relación aleatoria.

\begin{tabular}{|c|c|c|c|c|c|c|c|c|c|c|c|c|c|c|c|c|c|c|c|c|c|c|}
\hline & & AEN 1 & & & & GEN 2 & & & & EN 3 & & & & & GEI & & & & & & & \\
\hline 0 & & $\begin{array}{lll}1 & 1 & 1\end{array}$ & & 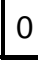 & 1 & 11 & & 0 & 0 & & & 0 & 1 & 1 & 0 & 0 & & & 0 & 0 & 1 & \\
\hline 0 & & 11 & 0 & 1 & 0 & 1 & 11 & 0 & 0 & 0 & 0 & 0 & 0 & 0 & 0 & 0 & & & ) & 0 & 0 & 1 \\
\hline 1 & & 1 & & 0 & & 0 & 1 & 0 & & & 1 & 0 & 0 & 0 & & & & & 0 & 0 & 1 & \\
\hline 0 & & 101 & 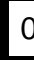 & 0 & & 01 & \begin{tabular}{l|l}
0 & 0 \\
\end{tabular} & 1 & 1 & 0 & 0 & 1 & 0 & 0 & & 0 & & & 0 & 0 & 0 & 0 \\
\hline 0 & & $\begin{array}{lll}0 & 1 & 0\end{array}$ & & & 1 & & 0 & & & & & & & 0 & & 1 & & & 0 & & & \\
\hline
\end{tabular}

Fig. 6: Muestra de la plantilla para la evaluación de los casos.

Tabla 3: Resultados del entrenamiento de la RNA

\begin{tabular}{|c|c|c|c|}
\hline & Muestras & $E M C$ & $R$ \\
\hline Entrenamiento & 1596 & $2,32981 \mathrm{E}-05$ & $9,90067 \mathrm{E}-01$ \\
\hline Validación & 342 & $2,62768 \mathrm{E}-05$ & $9,90233 \mathrm{E}-01$ \\
\hline Prueba & 342 & $1,98193 \mathrm{E}-05$ & $9,92804 \mathrm{E}-01$ \\
\hline
\end{tabular}




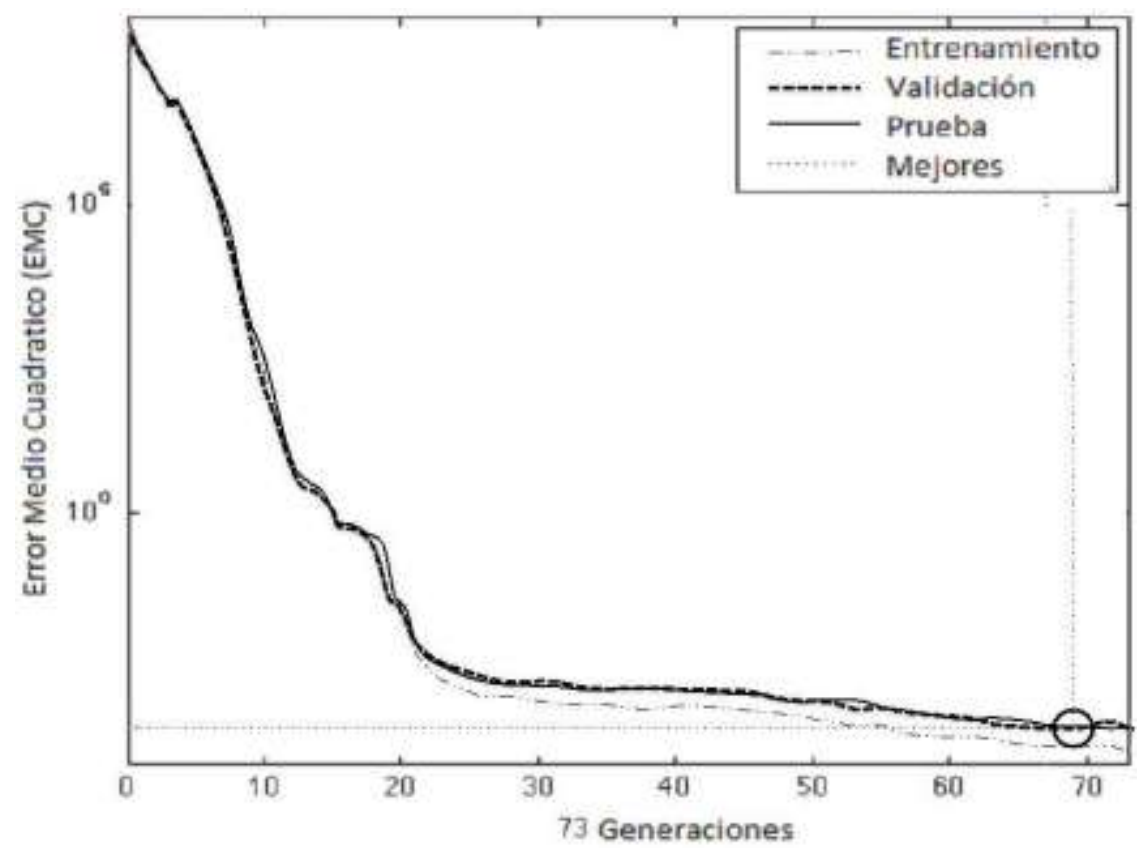

Fig. 7: Error medio cuadrático (EMC).

Una vez se entrenó la RNA para calcular la función objetivo, se realizaron pruebas para contrastar los resultados, calculando las pérdidas mediante un flujo de potencia convencional. En la Tabla 4 se presenta un resumen de los tiempos de cómputo para cada evaluación. Para esta simulación se utilizó un computador Intel Core 2 Duo de $2.53 \mathrm{GHz}$ con 8 GB de RAM. En este caso FO corresponde a la función objetivo usando un flujo de carga convencional y FO_RNA corresponde a la función objetivo calculada usando la Red Neuronal Artificial.

Tabla 4: Tiempo de computo AG y pérdidas del sistema

\begin{tabular}{|l|c|c|c|}
\hline & Tiempo $(\mathrm{s})$ & Pérdidas $(\mathrm{kW})$ & \% de disminución \\
\hline FO & $1.381,92$ & 656,25 & 20,68 \\
\hline FO_RNA & 326,51 & 666,86 & 19,5 \\
\hline
\end{tabular}

Se puede observar que la Red Neuronal Artificial ofrece una solución bastante aproximada a la solución real, con una desviación de 1,18\%; con la ventaja adicional de que una vez entrenada, puede estimar las pérdidas del sistema en menor tiempo que el flujo de carga convencional. En este sistema las pérdidas para el caso base, sin GD ascienden a $827,41 \mathrm{~kW}$. Al incorporar GD las pérdidas disminuyen en aproximadamente $20 \%$ (ver Tabla 4). La mejor solución obtenida con el AG se ilustra en la Figura 8 . En la Figura 9 se ilustra el perfil de tensiones de la red con y sin GD. Se puede observar que la GD además de contribuir a la reducción de pérdidas, mejora sustancialmente el perfil de tensiones de la red.

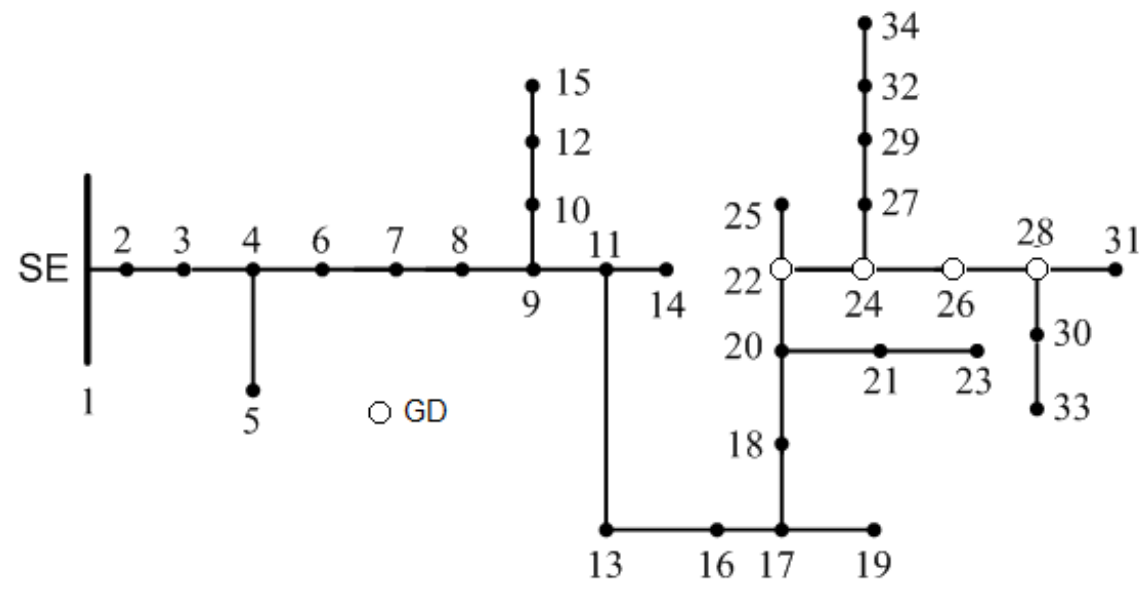

Fig. 8: Mejor configuración encontrada con la metodología propuesta. 


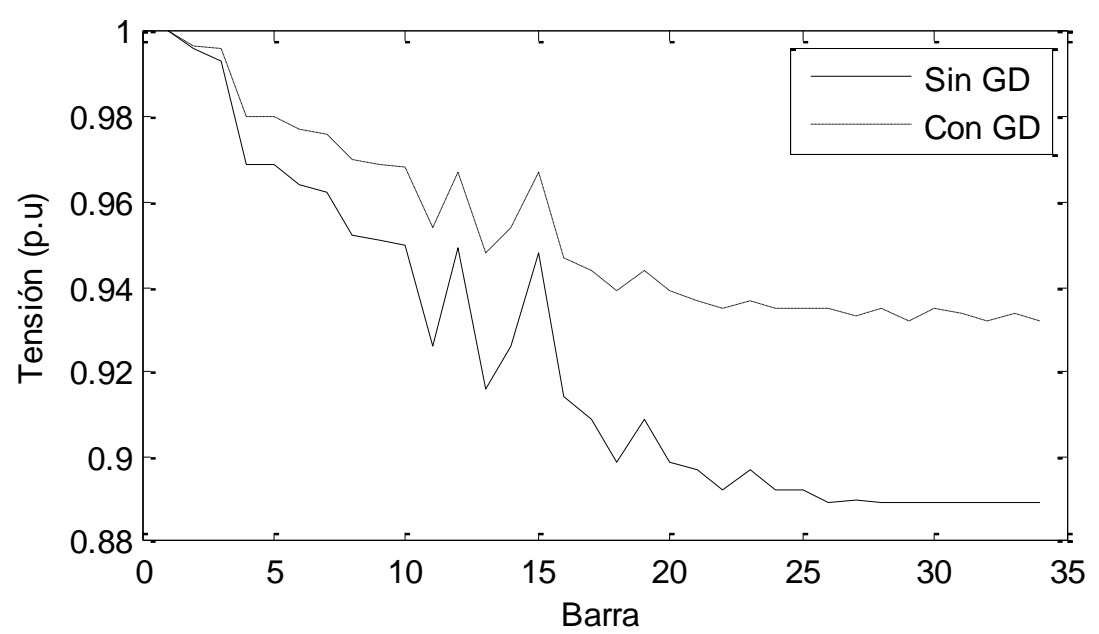

Fig.9: Perfil de tensiones con y sin GD.

\section{CONCLUSIONES}

En este artículo se presentó una metodología para la ubicación óptima GD en sistemas de distribución de energía eléctrica con el objetivo de minimizar pérdidas. La metodología propuesta consiste en un Algoritmo Genético Híbrido. Dicho algoritmo utiliza una Red Neuronal Artificial para la evaluación de la función objetivo y una búsqueda local. La metodología propuesta fue implementada con éxito en un sistema de 34 barras y muestra que se puede obtener una reducción considerable en las pérdidas del sistema gracias a una ubicación apropiada de la GD. Adicionalmente, se pudo comprobar que el uso de la RNA en la evaluación de la función objetivo permite mejorar los tiempos de cómputo del AG y focalizar los recursos en otros procesos de búsqueda.

\section{AGRADECIMIENTOS}

Los autores agradecen a la Universidad de Antioquia (UdeA) por el apoyo del proyecto "Sostenibilidad 20152016".

\section{REFERENCIAS}

Abou El-Ela, A.A., Allam, S.M. y Shatla, M.M., 2010. Maximal optimal benefits of distributed generation using genetic algorithms. Electric Power Systems Research, 80(7), pp.869-877 (2010)

Anaut, D., Di Mauro, G., Meschino, G., y Suarez, J., Optimización de Redes Eléctricas Mediante la Aplicación de Algoritmos Genéticos, Revista Inf. Tecnol. Cit: 20(4), 137-148 (2009)

Arroyave, L.B. y Lezama, J.L.,Valoración de los impactos técnicos de la generación distribuida en sistemas de energía eléctrica. Revista Tecnura, 17(1), pp.50-60 (2013)

Banerjee, B. y Islam, S.M., 2011. Reliability based optimum location of distributed generation. International Journal of Electrical Power \& Energy Systems, 33(8), pp.1470-1478 (2011).

Biswas, S., Goswami, S.K. y Chatterjee, A., Optimum distributed generation placement with voltage sag effect minimization. Energy Conversion and Management, 53(1), pp.163-174 (2012)

Borges, C.L.T. y Falcão, D.M., Optimal distributed generation allocation for reliability, losses, and voltage improvement. International Journal of Electrical Power \& Energy Systems, 28(6), pp.413-420 (2006)

García, C., García, E., y F. Villada., Algoritmo evolutivo eficiente aplicado a la planeacion de la expansion de sistemas de distribucion. Revista Inf. Tecnol. Cit: 23(4), pp.3-10 (2012)

Gautam, D. y Mithulananthan, N., Optimal DG placement in deregulated electricity market. Electric Power Systems Research, 77(12), pp.1627-1636 (2007) 
Hemdan, N.G. y Kurrat, M., Efficient integration of distributed generation for meeting the increased load demand. International Journal of Electrical Power \& Energy Systems, 33(9), pp.1572-1583 (2011)

Hung, D., Analytical expressions for DG allocation in primary distribution networks. IEEE Transactions on Energy Conversion, 25(3), pp.814-820 (2010)

Jabr, R.A. y Pal, B.C.,Ordinal optimisation approach for locating and sizing of distributed generation. IET Generation, Transmission \& Distribution, 3(8), pp.713-723 (2009)

Kang, y otros cuatro autores, Group search optimizer based optimal location and capacity of distributed generations. Neurocomputing, 78(1), pp.55-63 (2012)

Khalesi, N., Rezaei, N. y Haghifam, M.-R., 2011. DG allocation with application of dynamic programming for loss reduction and reliability improvement. International Journal of Electrical Power \& Energy Systems, 33(2), pp.288-295 (2011)

Khan, H. y Choudhry, M.A.,Implementation of Distributed Generation (IDG) algorithm for performance enhancement of distribution feeder under extreme load growth. International Journal of Electrical Power \& Energy Systems, 32(9), pp.985-997(2010)

Liu, Z., Wen, F. y Ledwich, G., Optimal Siting and Sizing of Distributed Generators in Distribution Systems Considering Uncertainties. Power Delivery, IEEE Transactions on, 26(4), pp.2541-2551 (2011)

López-Lezama, J.M., Contreras, J. y Padilha-Feltrin, A.,Location and contract pricing of distributed generation using a genetic algorithm. International Journal of Electrical Power \& Energy Systems, 36(1), pp.117-126 (2012)

Narvaez, P., Modelo para la selección de la ubicación y dimensionamiento de generación distribuida en sistemas eléctricos, Tesis de Maestria, Facultad de Ingeniería, Universidad de Antioquia (UdeA), Medellín, Colombia (2014).

Ouyang, W. y otros tres autores, Evaluation of distributed generation connecting to distribution network based on long-run incremental cost. IET Generation, Transmission \& Distribution, 5(5), pp.561-568 (2011)

Porkar, S. y otros tres autores, A novel optimal distribution system planning framework implementing distributed generation in a deregulated electricity market. Electric Power Systems Research, 80(7), pp.828$837(2010)$

Zimmerman, R., MATPOWER: Steady-state operations, planning, and analysis tools for power systems research and education. IEEE Transactions on Power Systems, 26(1), pp.12-19 (2011) 
